\title{
Effects of a Personalized Music Intervention for Persons with Dementia and their Caregivers
}

\author{
John Bufalini ${ }^{\mathrm{a}, *}$, Paul Eslinger ${ }^{\mathrm{b}}$, Erik Lehman $^{\mathrm{c}}$ and Daniel R. George ${ }^{\mathrm{d}}$ \\ ${ }^{a}$ Penn State College of Medicine, Hershey, PA, USA \\ ${ }^{\mathrm{b}}$ Departments of Neurology, Neural \& Behavioral Sciences, and Public Health Sciences, Penn State College of \\ Medicine, Hershey, PA, USA \\ ${ }^{\mathrm{c}}$ Department of Public Health Sciences, Penn State College of Medicine, Hershey, PA, USA \\ ${ }^{\mathrm{d}}$ Department of Humanities, Penn State College of Medicine, Hershey, PA, USA
}

Received 14 September 2021

Accepted 12 January 2022

Pre-press 25 January 2022

Published 2 February 2022

\begin{abstract}
.
Background: Given the challenges of developing disease-modifying treatments for Alzheimer's disease and related disorders, non-pharmacological interventions represent an increasingly promising approach in long-term care settings. Music-based interventions have been effective in improving the quality of life by influencing biopsychosocial factors that play a role in the progression of illnesses such as depression and anxiety. However, approaches have tended to focus exclusively on the person with dementia rather than integrating caregivers.

Objective: This study aimed to determine the impact of a music-based intervention on the quality of life of persons with dementia and their caregivers.

Methods: A mixed-methods study was conducted with seven dyads consisting of residents (aged 76-92) with diagnoses of dementia and their caregivers (aged 53-84) at a skilled nursing facility in Pennsylvania. Eight music intervention sessions were completed in the presence of the resident and caregiver using personalized playlists created for the dyad. Pre- and postintervention questionnaires were administered during each session, and observational data for both residents and caregivers were collected.

Results: Caregivers reported feeling less overwhelmed after the intervention with a mean difference of $-0.24 \pm 0.14, p=0.016$. Mean difference in the other 5 responses showed that listening to music had a beneficial impact for resident/caregiver dyads. Observations of interpersonal behavior supported the benefit of the intervention for these dyads.

Conclusion: Quantitative analysis of a personalized music intervention for residents/caregivers showed positive trends in increasing personal connection, and qualitative data identified greater appreciation of the relationship and increased bonding.
\end{abstract}

Keywords: Arts, caregivers, dementia, memory disorder, music

\footnotetext{
${ }^{*}$ Correspondence to: John A. Bufalini, MD, Penn State College of Medicine, Hershey, PA 17033, USA. Tel.: +1 7175318778 ;

E-mail: jbufalini@pennstatehealth.psu.edu.
} 


\section{INTRODUCTION}

Given the challenges of finding an effective treatment for Alzheimer's disease and related disorders, non-pharmacological methods represent an increasingly promising approach in caring for persons living with dementia. Approaches from the arts, including music therapy, have gained greater recognition in recent years [1-4]. While such interventions may not reverse the underlying biological progression of the illness, music has been shown to improve quality of life while addressing psychosocial factors like anxiety and depression that can accelerate progression [5]. In addition, music has been shown to increase both the verbal and behavioral understanding of situations in those with dementia [6]. Music-based interventions are generally safe, affordable, and reliable, and thus have become an increasingly well-regarded strategy used in residential care settings $[1,3]$.

As depicted in popular films such as the documentary Alive Inside (2014), researchers have begun to use personalized music approaches as a way to connect with those living with dementia [7]. Multiple studies have attempted to develop personalized playlists based on the past musical predilections of research participants to further enhance quality of life and well-being for those impacted [8-10], while other studies have established benefits for supporting tasks of daily living (e.g., taking a bath) [11]. The theoretical underpinning for the use of personalized playlists is that by utilizing music that naturally connects with the positive associations from one's past, it may help bring memories into active consciousness that have been rendered inactive by advancing dementia. Such memories may be a conduit to positive emotions and thus provide benefit to those listening.

However, studies evaluating the therapeutic effects of personalized music interventions have tended to focus solely on the person with dementia and not their caregiver [12], even though caregiver burden remains a significant concern [13-15]. Although studies have shown that counseling and support resources improve the well-being of caregivers, relatively little attention has been given to this demographic, despite their growing ranks in an aging world [16]. Caregivers tend to be close family members (at least initially in the progression of the illness), and this presents an opportunity for music to create a shared experience beneficial to both parties. Therefore, this pilot study sought to design and evaluate a personalized musicbased intervention involving both residents living with dementia and their caregivers. We hypothesized that sessions of personalized music would lead to increased interpersonal behaviors between residents and caregivers and greater sense of well-being.

\section{METHODS}

\section{Participants}

Seven dyads (i.e., residents living with dementia and their caregiver) were recruited from a skilled nursing facility in Hershey, Pennsylvania. The age range of residents was 76-92 years of age, with five female and two male residents participating in the study. Caregiver age ranged from 53 to 84 , with five female and two male caregivers participating in the study. All participants and caregivers identified their ethnicity as Caucasian. At the time of the study, all 7 residents had a clinical diagnosis of probable Alzheimer's disease or a related dementia. Written informed consent was obtained by the legal appointed representative of the resident and also for each of the caregivers. All 7 dyads completed all sessions and pre-post evaluations. All aspects of the study were approved by the Institutional Review Board at Pennsylvania State College of Medicine.

\section{Study design}

The study design included ten overall meetings. During the first session, basic demographic data and background information were obtained about each participant including clinical diagnosis, as well as history and preferences necessary to build personalized music lists (e.g., preferred genre, artist preference, and/or particular song requests). Personalization of the playlists was important. It first reduced the risk of unknowingly eliciting negative emotion associated with the music listening experience. Additionally, playing music that was meaningful to the dyad afforded greater potential for interest and engagement throughout the study. Furthermore, there is data to suggest that personalization of playlists (i.e., listening to familiar music from one's past) may result in specific beneficial structural and functional changes within individual's brain- particularly persons living with memory loss [17].

Individualized playlists were created for each dyad using the Apple Music's subscription streaming service, and the same selections were played in identical order during each session. Playlists were designed to last approximately 15 minutes for each dyad to 
Table 1

Questionnaire Use with Dyads During Intervention Session

\begin{tabular}{|c|c|}
\hline Pre-Intervention Questionnaire & Post-Intervention Questionnaire \\
\hline 1. Do you feel upset about the care of the resident? & 1. Have you felt upset currently about the care of the resident? \\
\hline $\begin{array}{l}\text { 2. Do you feel upset regarding the progression/condition of the } \\
\text { resident? }\end{array}$ & $\begin{array}{l}\text { 2. Do you feel upset regarding the progression/condition of the } \\
\text { resident? }\end{array}$ \\
\hline 3. Do you feel useful/important to the resident? & 3. Do you feel useful/important to the resident? \\
\hline 4. Do you feel overwhelmed regarding the resident's situation? & $\begin{array}{l}\text { 4. Do you feel overwhelmed currently regarding the resident's } \\
\text { situation? }\end{array}$ \\
\hline $\begin{array}{l}\text { 5. Do you feel that this session will have a positive effect for the } \\
\text { resident? }\end{array}$ & 5. Do you feel that this session had a positive effect on the resident? \\
\hline 6. Do you feel that this session will have a positive effect for you? & 6. Do you feel that this session had a positive effect on you? \\
\hline
\end{tabular}

provide standardization among the music experience not only among each dyad, but also throughout the entire study. The structure of each playlist consisted of five pieces of music that were readily available to the public. This standardization was undertaken to increase likelihood of replication at other skilled nursing facilities.

Subsequent to the introductory meeting, eight intervention sessions were conducted in a private gathering room to ensure a comfortable location for both resident and caregiver. Prior to each session, caregivers completed a questionnaire expressing their feelings regarding the upcoming music intervention (Table 1). These questions were scored on a 5-point Likert scale ranging from "Not at All" to "Extremely".

Following the completion of this questionnaire, the personalized playlist was then played for approximately 15 minutes with both the resident and caregiver in the same space. During each session, observational data regarding interpersonal behaviors between the resident and caregiver was recorded by one researcher (JB). Behaviors included: eye contact, physical touch, smiling, relaxed breathing, relaxed posture, leaning towards one another, and positive verbal communication. This assessment was undertaken using a modified instrument based on the Greater Cincinnati Chapter Well-Being Observation Tool [18]. At the conclusion of the session, caregivers were asked to complete the post-intervention questionnaire regarding how the intervention impacted them and their loved one. Similar questions were used in both pre- and post-settings (Table 1). This same format was implemented at each of the eight sessions for the duration of the project.

A final session was held at the end of the study in which participants were given an opportunity to express their thoughts regarding the music intervention. Additionally, a plan was made for the continuation of the music in a non-study capacity if desired by the participants. All sessions were completed between April 2018 and December 2018.

\section{Intervention}

Music was played from a research team member's (JB) iPhone in a private gathering room for the duration of the personalized playlist. Music for the playlist was downloaded from the Apple Music streaming service. A portable speaker that connected to the iPhone was placed on a table near residents and caregivers. Sessions spanned approximately 5 prechosen songs. One member of the research team (JB) was present for the entirety of each session. If the resident and/or caregiver felt that a session needed to be paused, the music was stopped until all felt it was appropriate to continue, and if the determination was made that no further intervention could occur for the day, then the session was concluded and rescheduled for a later date.

\section{Analysis}

Quantitative data was evaluated using the Wilcoxon Signed Rank Test to assess the difference in pre- and post-intervention means amongst all 7 dyads for each of 6 questions used. These means represent the collective responses pre vs post over the 8 intervention sessions. Qualitative data was also collected during each music session using overt observation and recorded using the modified instrument described above. A significance level of 0.05 was used for all comparisons, and all analyses were performed using SAS version 9.4 (SAS Institute, Cary, NC).

\section{RESULTS}

Pre-post analysis of the overall group mean for all 7 dyads showed that after listening to music, caregivers felt more positive and optimistic than before 
Table 2

Differences in Pre and Post Intervention Questionnaire Responses

\begin{tabular}{|c|c|c|c|c|}
\hline Question \# (question abbreviation) ${ }^{\mathrm{a}}$ & $\begin{array}{l}\text { Pre-Intervention } \\
\text { Mean }(N \pm \mathrm{SD})\end{array}$ & $\begin{array}{c}\text { Post-Intervention } \\
\text { Mean }(N \pm \mathrm{SD})\end{array}$ & $\begin{array}{c}\text { Mean Difference } \\
(N \pm \mathrm{SD})\end{array}$ & $p$ \\
\hline 1 (care of resident) & $0.96 \pm 0.88$ & $1.02 \pm 0.97$ & $0.06 \pm 0.20$ & 0.563 \\
\hline 2 (progression of resident) & $1.76 \pm 1.01$ & $1.61 \pm 0.96$ & $-0.15 \pm 0.30$ & 0.375 \\
\hline 3 (feel useful) & $3.21 \pm 0.52$ & $3.41 \pm 0.53$ & $0.20 \pm 0.41$ & 0.313 \\
\hline 4 (feel overwhelmed) & $1.79 \pm 1.01$ & $1.55 \pm 1.00$ & $-0.24 \pm 0.14$ & 0.016 \\
\hline 5 (effect on resident) & $3.21 \pm 0.62$ & $3.29 \pm 1.05$ & $0.16 \pm 0.51$ & 0.344 \\
\hline
\end{tabular}

p-values generated using a Wilcoxon Signed Rank Test. a'These variables were scored on a Likert Scale from 0 "Not at All” to 4 "Extremely."

the intervention (Table 2). Inquiries that assessed positive outcomes of the intervention (e.g., the caregiver feeling useful to the resident and two questions asking about the effect of the music) all showed upward trends following the music session. Furthermore, questions assessing negative emotions incorporated into caregiving such as feeling upset regarding progression of disease as well as feeling overwhelmed both decreased (Table 2). Although these 5 questionnaire responses showed directional changes that followed the hypothesized beneficial impact of music intervention, the changes found were not statistically significant. However, the question regarding caregivers feeling overwhelmed did yield a statistically significant decrease $(p=0.016)$.

Observations of interpersonal behavior consisted of recording the occurrence of these behaviors. The frequency of interpersonal behaviors in the 7 dyads over the 8 sessions conducted were: eye contact $85 \%-100 \%$, physical touch $43 \%-71 \%$, smiling $71 \%-100 \%$, relaxed breathing $14 \%-71 \%$, relaxed posture $71 \%-100 \%$, leaning closer $28 \%-57 \%$, and positive verbal communication $85 \%-100 \%$. Statistical analysis did not yield results that were significant or evident of measurable change between sessions in regard to the dyads' interpersonal behavior. Nonetheless, it was noted that resident and caregiver pairs varied in their responses; for instance, one resident went from sitting relatively quiet during the first intervention session to dancing during her last four, and a caregiver stated this was the first time she had seen her father smile in two years because of the music.

\section{DISCUSSION}

Our results suggest that personalized music interventions can have a positive impact for both residents and their caregivers. All but one question demonstrated a trend suggesting that the music intervention had engendered a benefit, which is clinically-if not statistically-significant. Such results are encouraging in that they show how, even in resource limited care settings, music can be a relatively low-cost, welltolerated intervention that can potentially improve quality of life for both residents and caregivers.

The limitations of this preliminary study include, most notably, a small sample size. Potential participants-particularly caregivers who did not live on-site and had to travel to the facility to participate-were deterred from enrolling by the criteria of having to be present for every intervention session. In addition, since participants were from only one institution, it is possible that those who participated may not represent the broader society as a whole in regards to their relationship with music, which can be influenced by factors such as ethnicity, class, and cultural and regional upbringing. Another limitation was that residents had observational data collected regarding their interaction with the music sessions that was not statistically measurable. Moreover, a researcher (JB) was present during all sessions, which could have biased the outcomes. Furthermore, the study's intervention was not conducted by a music therapist, but by one researcher (JB) as mentioned above. Although the lack of a formal music therapist to deliver the intervention was a limitation, it is also possible to view it as favorable to replication of this model at other resource-limited care settings.

Future studies could involve other individuals to take part in the music listening experience including grandchildren, other extended family members, or residential care staff, and could examine specific benefits for residents and caregivers in different contexts (e.g., activities of daily living, social and recreational activities, exercise, etc.). Effects may include behavioral and physiological factors (e.g., stress levels). As reflected in this study, mixed methods inquiry may be particularly effective in capturing the measurable and unmeasurable aspects of the intervention. Future studies could combine more rigorous statistical analysis with qualitative interviews of participants living 
with dementia and grounded analysis as a means of gathering rigorous data on the impact of the intervention on residents. Using questions in a yes-no format in both pre- and post-sessions could enable similar statistical analyses to be completed for all participants. More simple and straightforward questions such as "did you like the music?" or "do you want to listen again?" could be tracked over the duration of the project and afford the opportunity to specifically gain insight into how residents perceive the intervention.

Moreover, future use of a control group could offer greater statistical comparison to illustrate the impact of the music intervention. Studies have used group activity sessions to serve as a control with personalized music intervention [19], although it might also be beneficial to evaluate the use of personalized music intervention against non-personalized music intervention. Comparing personalized music interventions with non-musical arts-based activities might also be fruitful. Interpersonal behaviors could be tracked amongst these different environments so as to determine how personalization of the music impacts the experience for both the resident and caregiver. Moreover, if duration of interpersonal behaviors, instead of absolute occurrence was researched (as was the case in this study), change in each intervention type could be tracked over each session to provide more robust statistical analysis of the impact the intervention had on the dyad over time.

There remains a relatively small base of experimental data evaluating the effects of music interventions in long-term care settings. The advantages of this modality are that it is socially ubiquitous, can be personalized to individuals and caregivers, is generally cost-neutral, and presents minimal risk of injury (aside from the psychological distress that may be elicited by music with complex emotional associations). However, with proper screening and avoidance of potentially deleterious music, there would seem to be a high ceiling of potential benefit for use of personalized music in residential care both for those affected and caregivers alike.

\section{ACKNOWLEDGMENTS}

The project described was supported by the National Center for Advancing Translational Sciences, National Institutes of Health, through Grant TR002014. The content is solely the responsibility of the authors and does not necessarily represent the official views of the NIH.

\section{FUNDING}

This work was supported by the Joseph and Mary Caputo Research Award from Penn State College of Medicine Doctors Kienle Center for Humanistic Medicine.

\section{CONFLICT OF INTEREST}

The authors report no conflicts of interest.

\section{REFERENCES}

[1] Odell-Miller H (2021) Embedding music and music therapy in care pathways for people with dementia in the 21 st century-a position paper. Music Sci, doi:10.1177/ 20592043211020424

[2] Hanser SB (2021) Music-based interventions for people with Alzheimer's disease and related dementias: A review of the research. Music Med 13, 156-161.

[3] Clement-Cortes A, Hanser SB, Mercadal-Brotons M (2021) Foundations of dementia care for music therapy and music based interventions: Part 1. Music Med 13, doi: 10.47513/ mmd.v13i3.824.

[4] Livingston G, Kelly L, Lewis-Holmes E, Baio G, Morris S, Patel N, Omar RZ, Katona C, Cooper C (2014) A systematic review of the clinical effectiveness and cost-effectiveness of sensory, psychological and behavioural interventions for managing agitation in older adults with dementia. Health Technol Assess 18, 1-226, v-vi.

[5] Guétin S, Portet F, Picot MC, Pommié C, Messaoudi M, Djabelkir L, Olsen AL, Cano MM, Lecourt E, Touchon J (2009) Effect of music therapy on anxiety and depression in patients with Alzheimer's type dementia: Randomised, controlled study. Dement Geriatr Cogn Disord 28, 36-46.

[6] D'Aniello GE, Cammisuli DM, Cattaneo A, Manzoni GM, Molinari E, Castelnuovo G (2021) Effect of a music therapy intervention using Gerdner and colleagues' protocol for caregivers and elderly patients with dementia: A singleblind randomized controlled study. J Pers Med 11, 455.

[7] Rossato-Bennett M (2014) Alive Inside [Motion Picture]. Projector Media, USA.

[8] Garrido S, Stevens CJ, Chang E, Dunne L, Perz J (2019) Musical features and affective responses to personalized playlists in people with probable dementia. Am J Alzheimers Dis Other Demen 34, 247-253.

[9] Raglio A, Bellandi D, Baiardi P, Gianotti M, Ubezio MC, Zanacchi E, Granieri E, Imbriani M, Stramba-Badiale M (2015) Effect of active music therapy and individualized listening to music on dementia: A multicenter randomized controlled trial. J Am Geriatr Soc 63, 1534-1539.

[10] Garrido S, Stevens CJ, Chang E, Dunne L, Perz J (2018) Music and dementia: Individual differences in response to personalized playlists. J Alzheimers Dis 64, 933-941.

[11] Ray KD, Fitzsimmons S (2014) Music-assisted bathing: Making shower time easier for people with dementia. $J$ Gerontol Nurs 40, 9-13.

[12] Steen JVD, Soest-Poortvliet MV, Wouden HVD, Bruinsma M, Scholten R, Vonk A (2017) Music-based therapeutic interventions for people with dementia. Cochrane Database Syst Rev Music 5, 1-77. 
[13] Connors MH, Seeher K, Teixeira-Pinto A, Woodward M, Ames D, Brodaty H (2020) Dementia and caregiver burden: A three-year longitudinal study. Int J Geriatr Psychiatry 35, 250-258.

[14] Chiao CY, Wu HS, Hsiao CY (2015) Caregiver burden for informal caregivers of patients with dementia: A systematic review. Int Nurs Rev 62, 340-350.

[15] van den Kieboom R, Snaphaan L, Mark R, Bongers I (2020) The trajectory of caregiver burden and risk factors in dementia progression: A systematic review. J Alzheimers Dis 77, 1107-1115.

[16] Nguyen KV (2018) Special issue: Alzheimer's disease. AIMS Neurosci 5, 74-80.
[17] Fischer CE, Churchill N, Leggieri M, Vuong V, Tau M, Fornazzari LR, Thaut MH, Schweizer TA (2021) Long-known music exposure effects on brain imaging and cognition in early-stage cognitive decline: A pilot study. J Alzheimers Dis 84, 819-833.

[18] Kinney JM, Rentz CA (2005) Observed well-being among individuals with dementia: Memories in the Making, an art program, versus other structured activity. Am J Alzheimers Dis 20, 220-227.

[19] Ihara ES, Tompkins CJ, Inoue M, Sonneman S (2019) Results from a person-centered music intervention for individuals living with dementia. Geriatr Gerontol Int 19, 30-34. 